Korean J. Math. 20 (2012), No. 1, pp. 47-60

\title{
ANALYTIC CALCULATION OF EUROPEAN OPTION PRICING IN STOCHASTIC VOLATILITY ASSET MODEL
}

\author{
JAE-PILL OH
}

\begin{abstract}
We deal some analytic calculations for European option pricing by using the theory of elementary solution of generalized diffusion equation mainly.
\end{abstract}

\section{Introduction}

In this paper, we introduce a method of option pricing for several asset models which are special forms of a general class of volatility asset models.

In preprint [6], we can meet the general class of volatility asset models of the form

$$
\begin{aligned}
d S_{t} / S_{t} & =\mu d t+f\left(\sigma_{t}\right)\left[\sqrt{1-\rho^{2}} d W_{t}^{(1)}+\rho d W_{t}^{(2)}\right] \\
d \sigma_{t} / \sigma_{t} & =\beta\left(\sigma_{t}\right) d t+g\left(\sigma_{t}\right) d W_{t}^{(2)},
\end{aligned}
$$

where $W_{t}^{(1)}$ and $W_{t}^{(2)}$ are standard Brownian motions on a same probability space $(\Omega, \mathbf{F}, P)$. $S_{t}$ denotes the price of the (traded) asset and $\sigma_{t}$ is the (non-traded) stochastic local return variance at time $t$. The authors in [6] classified many stochastic volatility models by using some specifications and studied hedge strategies from an experimental as well as from an empirical perspective. Because the solutions of above stochastic differential equations can be represented by closed forms, perhaps we can get option pricing of asset models which are defined from above equations by same method. But, in this paper, we introduce some analytic

Received December 28, 2011. Revised February 27, 2012. Accepted March 6, 2012 .

2010 Mathematics Subject Classification: 60H10, 60J60, 62P20.

Key words and phrases: SDE, volatility asset model, diffusion process, elementary solution of diffusion equation, European option pricing. 
calculations of option pricing by using the theory of elementary solution of diffusion equation.

There are many asset models which are modified by stochastic volatilities. As we know, Black-Scholes volatility asset model is defined by two stochastic differential equations of the forms

$$
\begin{aligned}
d S_{t} & =S_{t}\left(\mu d t+\sigma_{t} d W_{t}\right), \\
d \sigma_{t} & =b\left(\sigma_{t}\right) d t+a\left(\sigma_{t}\right) d \bar{W}_{t},
\end{aligned}
$$

where $\mu$ is a constant, $W_{t}$ and $\bar{W}_{t}$ are independent Brownian motions, and $\sigma_{t}$ defined by (4) is volatility of asset price $S_{t}$ at time $t$. As we know, some stochastic volatility models $\sigma_{t}, t \geq 0$ defined by the solutions of stochastic differential equations are diffusion processes.

Stein and Stein $([8])$ introduced a mean reverting diffusion process

$$
d \sigma_{t}=-\delta\left(\sigma_{t}-\theta\right) d t+k d \bar{W}_{t},
$$

and used a Fourier inverse transformations to integrate the resulting partial differential equation for the price of a European option. Also, this model is a special form of above general class represented by (1) and (2). But this volatility model is a diffusion process, more in full, a Ornstein-Uhlenbeck process. Thus, we can use the elementary solution theory to get option prices.

We denote the density function $f_{W_{T}}$ of random variable $W_{T}$ of (3). Then we can get the European option pricing at time $t=0$ with maturity $T$, if it is call and $S_{T} \geq K$,

$$
\begin{aligned}
u(t, 0)= & e^{-r T} S_{0} e^{\mu T} \int_{R^{+}} \int_{0}^{\infty} h(z) p(T, s(x), s(z)) d m(s(z)) f_{W_{T}}(y) d y \\
& -e^{-r T} K .
\end{aligned}
$$

for the function $h(z)=\exp \left\{-(1 / 2) z^{2} T+z y\right\}$.

Under some special case, we can get the distribution function

$$
p(T, s(x), s(z)) \text {. }
$$

If we assume $b(x)=-b$ (constant) and in (4), this corresponding diffusion equation is periodic and we get

$$
p(t, x, y)=(1 / 2 \sqrt{\pi} t) \exp \left\{-\lambda_{0} t-(x-y)^{2} / 4 t+b(x+y) / 2\right\} .
$$

Under some reflecting boundary conditions, we get for large $t$,

$$
p(t, x, y) \sim(2 \pi t)^{-1 / 2} e^{-B(0)}
$$


and

$$
p(t, x, y) \sim 1 / M, \quad \text { for some } \quad M
$$

Therefore, in these case, we get for large $t$,

$$
E\left[h\left(\sigma_{t}\right)\right] \sim(2 \pi t)^{-1 / 2} \int_{0}^{\infty} a(x)^{-1} h(x) e^{B(x)-B(0)} d x,
$$

and

$$
E\left[h\left(\sigma_{t}\right)\right] \sim M^{-1} 2 \int_{0}^{\infty} a(x)^{-1} h(x) e^{B(x)} d x
$$

respectively.

In Section 2, we deal the elementary solution $p(t, x, y)$ of the generalized diffusion equation. In Section 3, we introduce the calculation of the price of European call option for, so-called, the Stein/Stein volatility model.

\section{Distribution of diffusion process}

Let $a \in C^{1}\left(R^{+}\right), b \in C\left(R^{+}\right), R^{+}=(0, \infty)$ with $a(x)>0$ and $\left(\bar{W}_{t}, P\right)$ be a standard Brownian motion. Let $\sigma_{t}, t>0$ be the solution of stochastic differential equation (4);

$$
\sigma_{t}=\sigma_{0}+\int_{R} a\left(\sigma_{t}\right) d \bar{W}_{t}+\int_{R} b\left(\sigma_{t}\right) d t
$$

with initial condition $\sigma_{0}=x \in(0, \infty)$. Then we know that the solution $\sigma_{t}, t>0$ is a diffusion process with the generator

$$
\mathbf{L}=\frac{1}{2} a^{2}(x) \frac{d^{2}}{d x^{2}}+b(x) \frac{d}{d x} .
$$

Thus, by the differential equations theory in diffusion process, we get the scale function $s(x)$ and the speed density $d M(y):=m(y) d y$ with the natural scale $y=s(x)$ as following;

(8) $s(x)=\int_{1}^{x} a(z)^{-1} e^{-B(z)} d z, \quad x \in(0, \infty)$,

(9) $m(y)=2 \int_{1}^{s^{-1}(y)} a(z)^{-1} e^{B(z)} d z, \quad y=s(x) \in(s(0), s(\infty))$,

where

$$
B(z):=\int_{1}^{z} \frac{2 b(\xi)}{a(\xi)^{2}} d \xi
$$


Then the value of functional $E\left[h\left(\sigma_{t}\right)\right]$ is the expectation of $h\left(\sigma_{t}\right)$ with respect to the probability $P$;

$$
E\left[h\left(\sigma_{t}\right)\right]=\int_{0}^{\infty} h(z) p(t, s(x), s(z)) d m(s(z)),
$$

where $h$ is a smooth function and $\sigma_{t}$ is the solution of stochastic differential equation (6).

Let $S=\left(l_{1}, l_{2}\right)$ be an open interval in the sense of McKean(c.f. [4]) with $-\infty \leq l_{1}<0<l_{2} \leq \infty$ and $m(x)$ a real valued nontrivial right continuous nondecreasing function on it with $m(0)=0$. For a functional

$$
v(t, x)=E\left[h\left(\sigma_{t}\right) \mid \sigma_{0}=x\right],
$$

where $h$ is a smooth function on $R$ with polynomial growth order, we get the second order partial differential equation which is so-called the generalized diffusion equation;

$$
\begin{aligned}
\frac{\partial v(t, x)}{\partial t} & =\mathbf{A} v(t, x), \\
v(0, x) & =h(x),
\end{aligned}
$$

where

$$
\mathbf{A}=\frac{1}{2} a(x)^{2} \frac{\partial^{2}}{\partial x^{2}}+b(x) \frac{\partial}{\partial x}
$$

on the interval $S$. Further, from the elementary solution (the fundamental solution of (12)) $p(t, x, y)$ of the generalized diffusion equation, we get

$$
v(t, x)=\int_{R} h(y) p(t, x, y) d y
$$

To get the elementary solution $p(t, x, y)$ of (12) which is in (13) on the interval $S$, so-called in the McKean sense, we need several a little bit complex steps. For each $\alpha \in \mathbf{C}$ and $x \in S$, let $\varphi_{1}(x, \alpha)$ and $\varphi_{2}(x, \alpha)$ be the solution of the integral equations

$$
\begin{aligned}
& \varphi_{1}(x, \alpha)=1+\alpha \int_{0+}^{x+}(x-y) \varphi_{1}(x, \alpha) d m(y), \\
& \varphi_{2}(x, \alpha)=x+\alpha \int_{0+}^{x+}(x-y) \varphi_{2}(x, \alpha) d m(y),
\end{aligned}
$$


respectively. Then for each $\alpha>0$, there exist the limits

$$
\begin{aligned}
& h_{1}(\alpha)=-\lim _{x \downarrow l_{1}} \varphi_{2}(x, \alpha) / \varphi_{1}(x, \alpha), \\
& h_{2}(\alpha)=\lim _{x \uparrow l_{2}} \varphi_{2}(x, \alpha) / \varphi_{1}(x, \alpha) .
\end{aligned}
$$

We will use usual convention $1 / \infty=0,( \pm a) / 0= \pm \infty, \infty \pm a=\infty$, and $-\infty \pm a=-\infty$ for positive real number $a$. Define the function $h /(\alpha)$ by the equality

$$
1 / h(\alpha)=1 / h_{1}(\alpha)+1 / h_{2}(\alpha)
$$

and $u_{i}(x, \alpha), i=1,2, \alpha>0, x \in S$ by

$$
u_{i}(x, \alpha)=\varphi_{1}(x, \alpha)+(-1)^{i+1} \varphi_{2}(x, \alpha) / h_{i}(\alpha) .
$$

Then it is known that $u_{1}(x, \alpha)\left[u_{2}(x, \alpha)\right]$ is positive non-decreasing $[$ resp. non-increasing] in $x \in S$ with $u_{1}(0, \alpha)=u_{2}(0, \alpha)=1$. Let

$$
\begin{aligned}
& h_{11}(\alpha)=h(\alpha), \quad h_{22}(\alpha)=-\left(h_{1}(\alpha)+h_{2}(\alpha)\right)^{-1}, \\
& h_{12}(\alpha)=h_{21}(\alpha)=-h(\alpha) / h_{2}(\alpha) .
\end{aligned}
$$

Then it is seen that all these functions $h_{i j}(\alpha), i, j=1,2$ can be analytically continued to the exterior of the half line $(-\infty, 0]$ in the complex plane. The spectral measure $\sigma_{i j}(d \lambda), i, j=1,2$ are given by

$$
\sigma_{i j}\left(\left[\lambda_{1}, \lambda_{2}\right]\right)=\lim _{\epsilon \downarrow 0} \frac{1}{\pi} \int_{\lambda_{1}}^{\lambda_{2}} \operatorname{Im} h_{i j}(-\lambda-\sqrt{-1} \epsilon) d \lambda
$$

for all continuity points $\lambda_{1}<\lambda_{2}$. Then the matrix valued measure $\left[\sigma_{i j}(d \lambda)\right]_{i, j=1,2}$ is symmetric and nonnegative definite. Thus, we define the elementary solution $p(t, x, y)$ of the generalized diffusion equation (12) by

$$
p(t, x, y)=\sum_{i, j=1}^{2} \int_{0-}^{\infty} e^{-\lambda t} \varphi_{i}(x,-\lambda) \varphi_{j}(y,-\lambda) \sigma_{i j}(d \lambda),
$$

for $t>0$ and $x, y \in S$.

We call the generalized diffusion equation (12) is periodic if $\mathbf{A}$ in (12) denoted by $\mathbf{A}=d(d / d s) / d m$ satisfy following (a), (b) and (c);

(a) $s(x)$ defined by (8) is continuous and increasing function,

(b) $m(y)$ defined by (9) is non-trivial, right continuous and nondecreasing, 
(c) There is a positive $\rho$ such that

$$
\begin{aligned}
s(x+1)-s(y+1) & =\rho^{-1}(s(x)-s(y)), \\
m(x+1)-m(y+1) & =\rho^{-1}(m(x)-m(y)) .
\end{aligned}
$$

Then we get that the elementary solution $p(t, x, y)$ of periodic diffusion equation (12) has some asymptotic behaviors to some more simple values; for large $t>0$, and $x, y \in R$,

$$
p(t, x, y) \sim \alpha\left(x, y, \lambda_{0}\right) t^{-1 / 2} \exp \left(-\lambda_{0} t\right),
$$

where $\lambda_{0}$ is the principle eigenvalue of $\mathbf{A}$, and $\alpha\left(x, y, \lambda_{0}\right)$ is a positive constant depending on $x, y$ and $\lambda_{0}$.

\section{Calculation of price in European options}

As we know, the solution of stochastic differential equation (3) is a closed form as following;

$$
S_{t}=S_{0} \exp \left\{\mu t-\frac{1}{2} \sigma_{t}^{2} t+\sigma_{t} W_{t}\right\} .
$$

The price of European option is defined by

$$
u(t, x)=E\left[e^{-r(T-t)} g\left(S_{T}\right) \mid S_{t}=s_{t}\right] .
$$

where the function $g$ is the pay-off function and $T$ is the maturity. The option is call if $g(x)=(x-K)^{+}$, and is put if $g(x)=(K-x)^{+}$where $(x)^{+}=\max \{0, x\}$ and $K$ is the strike price.

If we use the general class of stochastic volatility model (1) and (2) for the Stein/Stein volatility model in [6], we get of the form

$$
\frac{d \sigma_{t}}{\sigma_{t}}=\frac{\delta\left(\theta-\sigma_{t}\right)}{\sigma_{t}} d t+\frac{k}{\sigma_{t}} d \bar{W}_{t} .
$$

Thus, we can get the closed form of solution;

$$
\sigma_{t}^{2}=\sigma_{0}^{2} \exp \left\{\int \frac{\delta\left(\theta-\sigma_{t}\right)}{\sigma_{t}} d t+\int\left(\frac{k}{\sigma_{t}}\right) d \bar{W}_{T}\right\} .
$$

Thus, from this random variable $\sigma_{T}^{2}$, the asset price $S_{T}$ at time $T$ is of the form;

$$
S_{T}=S_{0} \exp \left\{\mu T-\frac{1}{2} \sigma_{T}^{2} T+\sigma_{T} W_{T}\right\} .
$$


From the definition of option price, the price of European call option at time $t=0$ is calculated as

$$
\begin{aligned}
u(t, 0) & =E\left[e^{-r T} g\left(S_{T}\right) \mid S_{0}=s_{0}\right] \\
& =E\left[e^{-r T}\left(S_{T}-K\right)^{+} \mid S_{0}=s_{0}\right] \\
& =e^{-r T} E\left[S_{T} \mid S_{0}=s_{0}\right]-e^{-r T} K, \quad \text { if } \quad S_{T} \geq K \\
& =e^{-r T} S_{0} E\left[\exp \left\{\mu T-\frac{1}{2} \sigma_{T}^{2} T+\sigma_{T} W_{T}\right\}\right]-e^{-r T} K .
\end{aligned}
$$

If we assume Brownian motion $W_{t}$ has mean $\mu_{t}^{*}$ and variance $\sigma_{t}^{*}$, we get density function $f_{W_{T}}$ of random variable $W_{T}$. For the solution $\sigma_{t}$ of stochastic differential equation (5), we assume Brownian motion $\bar{W}_{t}$ which is independent with Brownian motion $W_{t}$, has mean $\bar{\mu}_{t}^{*}$ and variance $\bar{\sigma}_{t}^{*}$. Then, we get density function $f_{\bar{W}_{T}}$ of random variable $\bar{W}_{T}$. Thus, from equations (19) and (21), we get

$$
\begin{aligned}
u(t, 0)= & e^{-r T} s_{0} e^{\mu T} E\left[\operatorname { e x p } \left\{-\frac{1}{2} \exp \left\{\sigma _ { 0 } ^ { 2 } \operatorname { e x p } \left\{\int \frac{\delta\left(\theta-\sigma_{t}\right)}{\sigma_{t}} d t\right.\right.\right.\right. \\
& \left.\left.+\int\left(\frac{k}{\sigma_{t}}\right) d \bar{W}_{T}\right\}\right\} T+\exp \left\{\sigma _ { 0 } ^ { 2 } \operatorname { e x p } \left\{\int \frac{\delta\left(\theta-\sigma_{t}\right)}{\sigma_{t}} d t\right.\right. \\
& \left.\left.\left.\left.+\int\left(\frac{k}{\sigma_{t}}\right) d \bar{W}_{T}\right\}\right\} W_{t}\right\}\right]-e^{-r T} K .
\end{aligned}
$$

Thus, we can get the European call option price at $t=T$; if we know the density functions $f_{W_{T}}$ and $f_{\bar{W}_{T}}$.

Stein and Stein volatility model is a diffusion process of the form of solution of stochastic differential equation (5) having a form of (reflected) Ornstein-Uhlenbeck diffusion process. The solution of this stochastic differential equation is represented as an integral form;

$$
\sigma_{t}=\sigma_{0}+(-\delta) \int_{0}^{t}\left(\sigma_{s}-\theta\right) d s+\int_{0}^{t} k d \bar{W}_{s}
$$

To get the price of European option, we can use the expectation (10) of the random variable $h\left(\sigma_{T}\right)$ for the solution $\sigma_{t}$ of $(4)$;

$$
E\left[h\left(\sigma_{T}\right) \mid \sigma_{0}=x\right]=\int_{0}^{\infty} p(T, s(x), s(z)) h(z) d m(s(z)) .
$$


In here,

$$
\begin{aligned}
s(x) & =\int_{1}^{x} a(z)^{-1} e^{-B(z)} d z, \quad x \in(0, \infty) \\
& =\int_{1}^{x} \frac{1}{k} e^{-B(z)} d z,
\end{aligned}
$$

and

$$
\begin{aligned}
m(y) & =2 \int_{1}^{s_{1}^{-1}(y)} a(z)^{-1} e^{B(z)} d z \\
& =2 \int_{1}^{s_{1}^{-1}(y)} \frac{1}{k} e^{B(z)} d z, \quad y=s(x) \in(s(0), s(\infty)),
\end{aligned}
$$

where

$$
\begin{aligned}
B(z) & :=\int_{1}^{z} \frac{2 b(\xi)}{a(\xi)^{2}} d \xi=\int_{1}^{z} \frac{-2 \delta(\xi-\theta)}{k^{2}} d \xi \\
& =\frac{\delta}{k}\left(2 \theta z+1-\delta z^{2}-2 \delta \theta\right),
\end{aligned}
$$

because of $a(x)=k, b(x)=-\delta(x-\theta)$. From the fact $(21)$, if $S_{T} \geq K$, we get

$$
u(t, 0)=e^{-r T} s_{0} \exp \{\mu T\} E\left[\exp \left\{-\frac{1}{2} \sigma_{T}^{2} T+\sigma_{T} W_{T}\right\}\right]-e^{-r T} K,
$$

where the random variable $\sigma_{T}$ is the solution of (4) at $t=T$. Thus, we get the European option price for the function $h(z)=\exp \left\{-(1 / 2) z^{2} T+\right.$ $z y\}$,

$$
\begin{gathered}
u(t, 0)=e^{-r T} S_{0} e^{\mu T} \int_{R^{+}} \int_{0}^{\infty} h(z) p(T, s(x), s(z)) f_{W_{T}}(y) d y d m(s(z))-e^{-r T} K \\
=e^{-r T} S_{0} e^{\mu T} \int_{R^{+}} \int_{0}^{\infty}\left[\exp \left\{-\frac{1}{2} z^{2} T+z y\right\}\right] f_{W_{T}}(y) \\
p(T, s(x), s(z)) d m(s(z)) d y-e^{-r T} K,
\end{gathered}
$$

where $f_{W_{T}}(y)$ is the density function of $W_{T}$. To get calculation of $p(T, s(x), s(z))$, we will introduce several cases.

[I]. The following formulation of this subsection is found in [5] mainly. 
Let us assume $b(\cdot)=0$ in (4). From the stochastic differential equation (4), if we use $s(x)$ and $m(y)$ of (8) and (9), we get

$$
\begin{aligned}
s(x) & =\int^{x} 1 d \xi=x, \\
m(x) & =1 / \sigma^{2}(x) s^{\prime}(x)=1 / \sigma^{2}(x) .
\end{aligned}
$$

Then, for the generator $\mathbf{L}$ in (7) and the function $h$ in (10), we get

$$
\begin{aligned}
\mathbf{L} h(x) & =\frac{1}{2} a^{2}(x) \frac{d^{2}}{d x^{2}} h(x)+b(x) \frac{d}{d x} h(x) \\
& =\frac{1}{2} \frac{d}{d M} \frac{d}{d s} h(x)=\frac{1}{2} \sigma^{2}(x) h^{\prime \prime}(x) .
\end{aligned}
$$

Then we get that $\rho=1$ of (15) and (16). Therefore, in this case, we can use the theory of periodic diffusion equation easily.

As a more general case of periodic diffusion operator $\mathbf{A}$ in (12), if we think a generator

$$
\mathbf{L}=a^{2}(x) \frac{d^{2}}{d x^{2}}-b \frac{d}{d x},
$$

where $b$ is a real number, then we can get following;

$$
\rho=e^{b}, \quad d s(x)=e^{b x} d x, \quad d m(x)=e^{-b x} d x,
$$

satisfying (15) and (16). Therefore, as we see in [4] and [5], we get $S=\left[\lambda_{0}, \infty\right)$, $\lambda_{0}=b^{2} / 4$,

$$
\alpha\left(x, y, \lambda_{0}\right)=(1 / 2 \sqrt{\pi}) e^{b(x+y) / 2},
$$

and for $t>0, x, y \in R$,

$$
p(t, x, y)=(1 / 2 \sqrt{\pi} t) \exp \left\{-\lambda_{0} t-(x-y)^{2} / 4 t+b(x+y) / 2\right\} .
$$

From this elementary solution, we get option price by using (25). Further, if we know some real data, we can get the period of the movement of prices of asset and volatility. $[4]$.

The following formulation of this subsection [II] and [III] can be found in

[II]. From the stochastic differential equation (4), if we assume

$$
\begin{aligned}
& \text { (27) } \int_{0}^{1}\left|\frac{2 b(x)}{a(x)^{2}}\right| d x=\int_{0}^{1}\left|\frac{-2 \delta(x-\theta)}{k^{2}}\right| d x<\infty \\
& \text { (28) } \int_{0}^{1} a(x)^{-1} d x=\int_{0}^{1} \frac{1}{k} d x=\infty \\
& \text { (29) } \int_{1}^{\infty} a(x)^{-1} e^{B(x)} \int_{1}^{x} a(z)^{-1} e^{-B(z)} d z=\int_{1}^{\infty} \frac{1}{k} e^{B(x)} \int_{1}^{x} \frac{1}{k} e^{-B(z)} d z<\infty,
\end{aligned}
$$


and we impose the reflecting boundary condition at upper boundary $B_{1}$ in case of

$$
B_{1}:=\int_{1}^{\infty} a(x)^{-1} e^{-B(x)} d x<\infty,
$$

then we get $l_{1}=s(0+)=-\infty, l_{2}=\infty$, and

$$
\begin{aligned}
& m(y) \sim 2 e^{2 B(0)} y \text { as } y \rightarrow-\infty, \\
& \int_{0}^{\infty} y d m(y)<\infty .
\end{aligned}
$$

Thus, we get long time asymptotic behavior of the elementary solution $p(t, x, y)$ of the generalized diffusion equation as following

$$
p(t, x, y) \sim(2 \pi t)^{-1 / 2} e^{-B(0)} \quad \text { as } \quad t \rightarrow \infty .
$$

For the option pricing of asset price process $S_{t}, t \geq 0$, we write the expectation of $h\left(\sigma_{t}\right)$ with respect to probability measure $P$ as following

$$
\begin{aligned}
E\left[h\left(\sigma_{t}\right)\right] & =\int_{0}^{\infty} p(t, s(x), s(z)) h(z) d m(s(z)) \\
& \sim(2 \pi t)^{-1 / 2} \int_{0}^{\infty} a(x)^{-1} h(x) e^{B(x)-B(0)} d x \text { as } t \rightarrow \infty .
\end{aligned}
$$

for all $h$ such that the integral in the right-hand side converges absolutely.

[III]. Instead of (27), (28) and (29), if we assume

$$
2 \int_{0}^{\infty} a(x)^{-1} e^{B(x)} d x<\infty
$$

and impose the reflecting boundary condition at lower boundary $B_{2}$ in case of

$$
B_{2}:=-\int_{0}^{1} a(x)^{-1} e^{-B(x)} d x>-\infty,
$$

then we get $l_{1}=-\infty, l_{2}=\infty$ and

$$
M:=m(\infty)-m(-\infty)=2 \int_{0}^{\infty} a(x)^{-1} e^{B(x)} d x<\infty .
$$

Then, we get

$$
p(t, x, y) \sim 1 / M \quad \text { as } \quad t \rightarrow \infty .
$$

and

$$
E\left[h\left(\sigma_{t}\right)\right] \sim M^{-1} 2 \int_{0}^{\infty} a(x)^{-1} h(x) e^{B(x)} d x \quad \text { as } \quad t \rightarrow \infty
$$

for all $h$ such that the integral in the right-hand side converges absolutely. 


\section{Some other volatility asset models}

\section{Hull and White volatility model.}

From [3], Hull and White volatility model is defined by the solution $\sigma_{t}^{2}$ of the stochastic differential equation

$$
d \sigma_{t}^{2}=\sigma_{t}^{2}\left(p d t+q d \bar{W}_{t}\right)
$$

Thus, from the solution, we can get a non-negative random variable of the form;

$$
\sigma_{T}^{2}=\sigma_{0}^{2} \exp \left\{p T-\frac{1}{2}\left(q^{2} T\right)+q \bar{W}_{T}\right\} .
$$

From this random variable $\sigma_{T}^{2}$ and the asset price $S_{T}$ at time $T$, the price of European call option at time $t=0$ is calculated as (21).

If we assume the Brownian motion $W_{t}$ has the density function $f_{W_{T}}$ of random variable $W_{T}$. For the solution $\sigma_{t}$ of stochastic differential equation (34), we assume the Brownian motion $\bar{W}_{t}$ has the density function $f_{\bar{W}_{T}}$ of random variable $\bar{W}_{T}$. Then, we can calculate option price by following;

$$
\begin{aligned}
u(t, 0)= & e^{-r T} s_{0} e^{\mu T} E\left[\operatorname { e x p } \left\{-\frac{1}{2} \exp \left\{p T-\frac{1}{2}\left(q^{2} T\right)+q \bar{W}_{T}\right\} T\right.\right. \\
& \left.\left.\left.\quad+\exp \left\{p T-\frac{1}{2}\left(q^{2} T\right)+q \bar{W}_{T}\right\}^{\frac{1}{2}} W_{T}\right\}\right\}\right]-e^{-r T} K, \quad \text { if } \quad S_{T} \geq K .
\end{aligned}
$$

To calculate option price by using elementary solution of diffusion process, we rewrite (34) as

$$
\sigma_{t}=\sigma_{0}+\int_{0}^{\infty} q \sigma_{t} d \bar{W}_{t}+\int_{0}^{\infty} p \sigma_{t} d t, \quad \sigma_{t}>0
$$

with condition $\sigma_{0}=x>0$. Then we get generator

$$
\mathbf{L}=\frac{1}{2}(q x)^{2} \frac{d^{2}}{d x^{2}}+p x \frac{d}{d x},
$$

scale function $s(x)$, and speed density $m(x)$ for $x \in(0, \infty)$;

$$
\begin{aligned}
s(x) & =\int_{0}^{x} e^{-B(z)} d z=\int_{0}^{x} z^{-\frac{2 p}{q^{2}}} d z=\int_{1}^{x} z^{\frac{q^{2}}{2 p}} d z=\frac{2 p}{q^{2}+2 p} x^{\frac{q^{2}+2 p}{2 p}}, \\
m(x) & =1 /(q x)^{2} s^{\prime}(x)=1 /(q x)^{2} x^{\frac{q^{2}}{2 p}}, \quad x \in(0, \infty) .
\end{aligned}
$$

because

$$
B(z):=\int_{0}^{z} \frac{2 p \xi}{(q \xi)^{2}} d \xi=\int_{1}^{z} \frac{2 p}{q^{2}} \frac{1}{\xi} d \xi=\frac{2 p}{q^{2}} \ln z .
$$

To get the elementary solution $p(T, x, y)$, we can use the theory of diffusion equation. But, as we know, the solution of (34) is a geometric Brownian motion which is more simple than diffusion process in general to get option 
prices. Thus, it is more simple if we use (36) by using density functions of $W_{T}$ and $\bar{W}_{T}$.

\section{Heston volatility model.}

The Heston volatility model(c.f. [2]) is denoted by a stochastic differential equation having a solution of the form;

$$
\sigma_{t}^{2}=\sigma_{0}^{2}+\int_{0}^{t} \delta\left(\theta-\sigma_{s}^{2}\right) d s+\int_{0}^{t} k \sigma_{s} d \bar{W}_{s}
$$

As a similar method as the Stein/Stein model, if $W_{t}$ and $\bar{W}_{t}$ are independent, to get the price of European option we need to get the expectation of random variable $h\left(\sigma_{t}^{2}\right)$, where $\sigma_{t}^{2}$ is the solution of stochastic differential equation (37);

$$
E\left[h\left(\sigma_{t}^{2}\right) \mid \sigma_{0}=x\right]=\int_{0}^{\infty} p(t, s(x), s(z)) h(z) d m(s(z)) .
$$

In here,

$$
\begin{aligned}
s(x) & =\int_{1}^{x} a(z)^{-1} e^{-B(z)} d z, \quad x \in(0, \infty) \\
& =\int_{1}^{x} \frac{1}{k z^{1 / 2}} e^{-B(z)} d z, \quad x \in(0, \infty),
\end{aligned}
$$

and

$$
m(y)=2 \int_{1}^{s^{-1}(y)} \frac{1}{k z^{1 / 2}} e^{B(z)} d z, \quad y=s(x) \in(s(0), s(\infty)),
$$

where

$$
\begin{aligned}
B(z) & :=\int_{1}^{z} \frac{2 b(\xi)}{a(\xi)^{2}} d \xi=\int_{1}^{z} \frac{2 \delta(\theta-\xi)}{k^{2} \xi} d \xi \\
& =\frac{2 \delta \theta}{k^{2}}(\ln 2)-\frac{2 \delta}{k^{2}} z+\frac{2 \delta}{k^{2}},
\end{aligned}
$$

because of $a(x)=k z^{1 / 2}, b(x)=\delta(\theta-x)$. From the solution of (3), we get

$$
S_{T}=S_{0} \exp \left\{\mu T-\frac{1}{2} \sigma_{T}^{4} T+\sigma_{T}^{2} W_{T}\right\},
$$

and, if $S_{T} \geq K$, we get

$$
u(t, 0)=e^{-r T} s_{0} \exp \{\mu T\} E\left[\exp \left\{-\frac{1}{2} \sigma_{T}^{4} T+\sigma_{T} W_{T}\right\}\right]-e^{-r T} K,
$$

where the random variable $\sigma_{T}^{2}$ is the solution of (37) at $t=T$. Thus, we can get the European option price by using

$$
\begin{array}{r}
u(t, 0)=e^{-r T} S_{0} \exp \{\mu T\} \int_{R^{+}} \int_{-1}^{\infty}\left[\exp \left\{-\frac{1}{2} z^{4} T+z^{2} y\right\}\right] \\
f_{W_{T}}(y) p(T, s(x), s(z)) d m(s(z)) d y-e^{-r T} K,
\end{array}
$$


where $f_{W_{T}}(y)$ is the density function of $W_{T}$ in (3).

But, Heston volatility model has a big meaning when $W_{t}$ and $\bar{W}_{t}$ are not independent. In this case, we can get $\operatorname{Corr}\left(W_{t}, \bar{W}_{t}\right) \neq 0$ which discharge for the leverage works. Thus, to study leverage works, our diffusion method is needed not.

\section{Summary}

As we know from [6] and above, for many types of volatility asset models which are defined by the solutions of stochastic differential equations and are represented by closed forms, we can calculate option prices if we know the distribution of volatility $\sigma_{t}$ basically. For the types of volatility models represented by stochastic differential equations, if the solutions(volatility models) are diffusion processes, we can calculate the option prices by using the theory of diffusion equations, i.e., using the theory of elementary solutions of diffusion equations derived from some functionals. Further, if we impose some conditions to define various types of diffusion equations (c.f. [4] and [5]), we can get option prices for various types of volatility models which are defined by some diffusion processes.

\section{References}

[1] S. Herzel, Option pricing with stochastic volatility models, Decis. Econ. and Finance, 23 (2000), 73-99.

[2] S.L. Heston, A closed-form solution for options with stochastic volatility with applications to bond and currency options, Review of Financial Studies, 6 (1993), 327-343.

[3] J. Hull and A. White, The pricing of options on assets with stochastic volatilities, The Journal of Finance, 42 (2) (1987), 281-300.

[4] N. Minami, Y. Ogura and M. Tomisaki, Asymptotic behavior of elementary solutions of one-dimensional generalized diffusion equations, The Annals of Probability, 13 (3) (1985), 698-715.

[5] J. Oh and M. Tomisaki, Asymptotic behavior of elementary solutions of periodic generalized diffusion equations, The Journal of the Korean Mathematical Society, 25 (2) (1988), 175-198.

[6] R. Poulsen, K.R. Schenk-Hoppe and C.-O. Ewald, Risk minimization in stochastic volatility models: Model risk and empirical performance, preprint, www.math.ku.dk/ rolf/Klaus/pse.pdf.

[7] P. Protter, Stochastic integration and differential equations, Berlin Heidelberg N.Y., Springer, 2nd Printing, 1992.

[8] E.M. Stein and J.C. Stein, Stock price distributions with stochastic volatility: An analytic approach, Review of Financial Studies, 4 (1991), 727-752. 
Department of Mathematics

Kangweon National University

Chuncheon 200-701, Korea

E-mail: jpoh@kangwon.ac.kr 